\title{
L1 cell adhesion molecule (L1CAM) is a strong predictor for locoregional recurrences in cervical cancer
}

\author{
Marlies Schrevel ${ }^{1,2}$, Willem E. Corver ${ }^{1}$, Margit E. Vegter ${ }^{1}$, Natalja T. Ter Haar ${ }^{1}$, \\ Enno J. Dreef ${ }^{1}$, Jogchum J. Beltman ${ }^{2}$, Gemma Kenter ${ }^{3}$, Tjalling Bosse ${ }^{1}$, Cornelis D. \\ de Kroon ${ }^{2}$ and Ekaterina S. Jordanova ${ }^{1,3}$ \\ ${ }^{1}$ Department of Pathology, Leiden University Medical Center, Leiden, The Netherlands \\ ${ }^{2}$ Department of Gynecology, Leiden University Medical Center, Leiden, The Netherlands \\ ${ }^{3}$ Department of Gynecology, VUmc, Centre for Gynecologic Oncology, Amsterdam, The Netherlands \\ Correspondence to: Ekaterina S. Jordanova, email: E.S.Jordanova@lumc.nl \\ Keywords: cervical cancer, epithelial mesenchymal transition (EMT), Ll cell adhesion molecule (L ICAM), locoregional recurrence, \\ prognosis \\ Received: April 08, $2017 \quad$ Accepted: August 21, $2017 \quad$ Published: September 18, 2017 \\ Copyright: Schrevel et al. This is an open-access article distributed under the terms of the Creative Commons Attribution License \\ 3.0 (CC BY 3.0), which permits unrestricted use, distribution, and reproduction in any medium, provided the original author and \\ source are credited.
}

\section{ABSTRACT}

Background: L1 cell adhesion molecule (L1CAM) has been shown to be a prognostic marker in various cancer types, and has been suggested to play a role in epithelial mesenchymal transition (EMT). Here, we determined the prognostic significance of L1CAM in cervical cancer and its association with vimentin expression on tumor cells, indicative of EMT.

Methods: Formalin-fixed, paraffin-embedded primary tumor samples from $\mathbf{3 7 2}$ cervical cancer patients were collected for immunohistochemical analysis of L1CAM expression. In 109 FFPE specimens, the percentage of vimentin expressing tumor cells was determined by flow cytometry.

Results: Positive L1CAM expression ( $\geq 10 \%$ of tumor cells) was associated with disease-free survival, validated using RNAseq TCGA data. L1CAM expression was independently associated with locoregional recurrence-free survival (hazard ratio $2.62,95 \% \mathrm{CI} 1.33-5.17, P=0.006)$, and strongly associated with percentage of vimentin expressing tumor cells $(P=\mathbf{0 . 0 0 3})$. Expression of both L1CAM and vimentin indicated a subgroup with the highest risk of recurrence (hazard ratio $3.15,95 \% \mathrm{CI}$ $1.25-7.92, P=0.015$ ).

Conclusion: L1CAM might be a promising new prognostic marker for locoregional recurrences in cervical cancer, and its association with vimentin expression suggests that L1CAM might affect tumor aggressiveness, possibly through EMT.

\section{INTRODUCTION}

Cervical cancer is the fourth most common type of cancer among women worldwide [1]. Cervical cancer spreads in a progressive and predictable manner through regional lymphatics, suggesting that disease-recurrence is a result of insufficient primary treatment. For stage IB-IIA cervical cancer, the primary treatment is either primary radical surgery followed by adjuvant (chemo)radiotherapy, or primary chemoradiotherapy. Adjuvant radiotherapy after primary radical surgery is indicated when two out of three pathologic factors are present: vaso-invasion, tumor size $\geq 4 \mathrm{~cm}$, and tumor invasion $\geq 2 / 3$ or $\geq 15 \mathrm{~mm}$ (Sedlis criteria), but the risk of recurrences is only reduced by $\sim 50 \%[2,3]$. Aside from currently used pathologic factors, molecular tumor markers might be helpful in predicting disease-recurrence, thus improving the selection of patients requiring adjuvant treatment. 
A promising new marker is L1 cell adhesion molecule (L1CAM) [4]. L1CAM was first reported to be a strong prognostic factor for metastases in cutaneous malignant melanoma [5]. A recent meta-analysis reviewed 37 studies on the association between L1CAM expression and survival parameters [6]. L1CAM was shown to be a prognostic factor for overall survival in colorectal cancer, ovarian cancer, neuroendocrine tumors, GIST, cholangiocarcinoma, renal cell cancer, non-small cell lung cancer (NSCLC), hepatocellular cancer, endometrial cancer and for disease-free survival in neuroblastoma, ovarian cancer, neuroendocrine tumors, gallbladder cancer, hepatocellular cancer and endometrial cancer. The most informative cut-off value in these different tumor types was $>10 \%$ of L1CAM positive tumor cells, as determined by immunohistochemistry [6].

One suggested mechanism through which L1CAM influences tumor progression and metastasis formation is epithelial-mesenchymal transition (EMT) [7]. During this process, epithelial cells loose cell to cell junctions and apico-basal polarity, resulting in a migratory and invasive mesenchymal-like phenotype [4]. Zecchini et al., describe a dual role for L1CAM, as L1CAM supported cell-cell adhesion and enhanced apoptosis in nontransformed ovarian epithelial cells, while it inhibited cell-cell adhesion and apoptosis and promoted malignancyrelated properties, such as cell proliferation, invasion, and transendothelial migration in ovarian carcinoma cells [8]. In NSCLC, L1CAM expression was positively correlated with vimentin, beta-catenin, and slug expression, but inversely correlated with E-cadherin expression [9]. In cervical cancer, human papilloma virus (HPV) oncogene E6 has been shown to induce a mesenchymal phenotype in a chemoresistant cervical cancer cell line ( $\mathrm{SiHaCR})$, with elevated levels of survivin, snail, slug, twist and vimentin and reduced levels of E-cadherin [10]. In cetuximab/ chemotherapy-treated NSCLC, vimentin expression was significantly associated with shorter progression-free survival [11]. Similarly, in oral squamous cell carcinoma, vimentin expression was associated with lymph node metastasis and poor overall survival [12].

The aim of the present study was to determine the protein expression of L1CAM and its association with clinical parameters and patient survival in a large cohort of
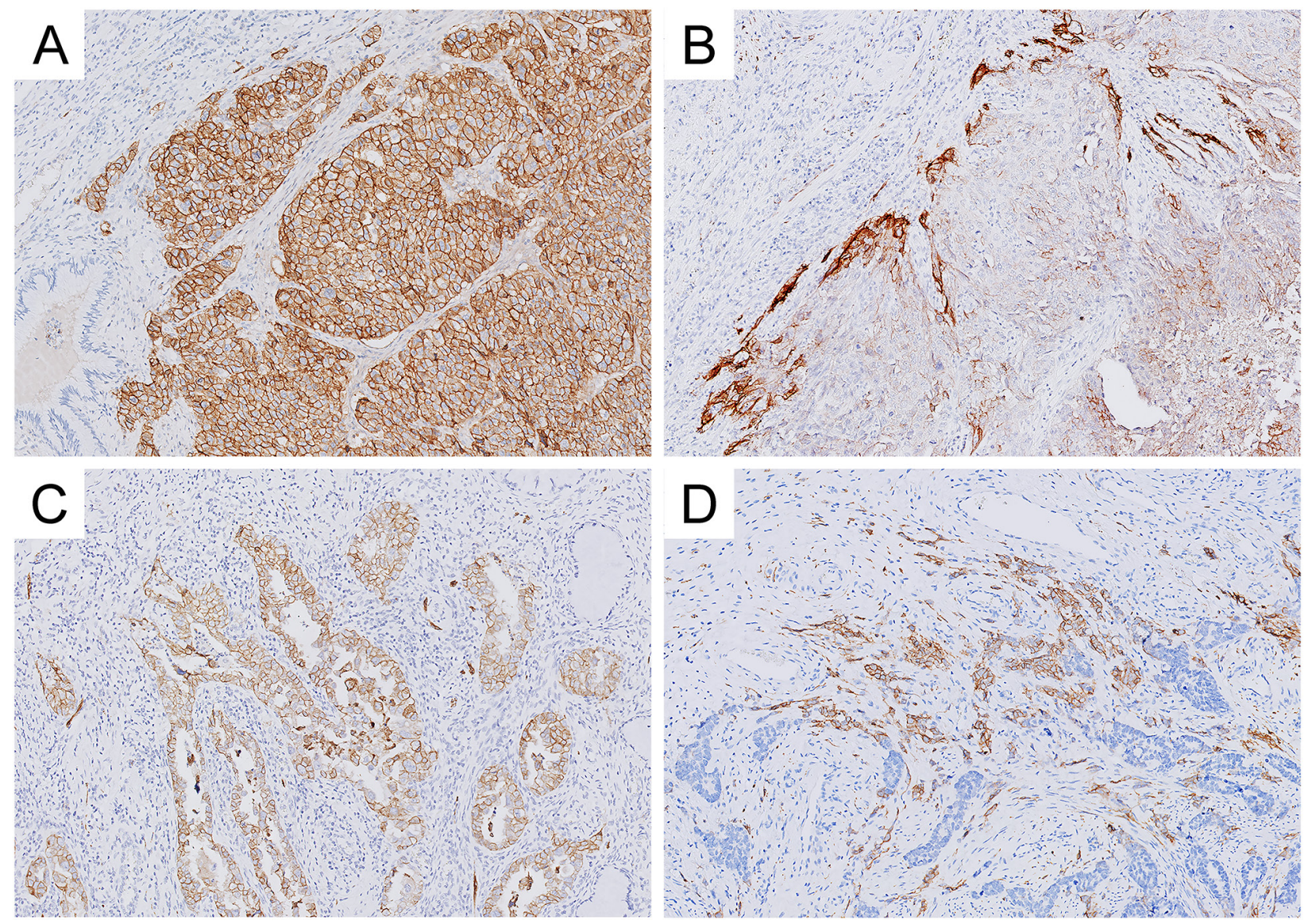

Figure 1: Representative examples of L1 cell adhesion molecule (L1CAM) expression in cervical carcinoma. (A) diffuse staining pattern and (B) infiltration border positivity in squamous cell carcinoma, (C) diffuse staining pattern and (D) infiltration border positivity in adenocarcinoma. 
cervical cancer patients, as well as in a TCGA validation cohort. Furthermore, we determined the association between L1CAM expression and percentage of vimentin expressing tumor cells, as a marker for EMT.

\section{RESULTS}

\section{L1CAM expression in relation to patient characteristics}

Immunohistochemical staining of L1CAM in cervical cancer tissues was observed to be mainly membranous, accompanied in some cases by weak cytoplasmic staining. Samples with staining in less than $10 \%$ of the tumor cells, or samples with no L1CAM staining, were considered negative ( $\mathrm{N}=292,79 \%)$. In 80 cases $(21 \%)$, more than $10 \%$ of the tumor cells were L1CAM positive, of which 35 cases ( $9 \%$ ) showed staining in more than $50 \%$ of the tumor. Of the L1CAM positive cases $(\geq 10 \%)$, positive staining was observed exclusively at the infiltrating front in $42 \%$ of cases, while $34 \%$ showed a diffuse staining, including the infiltrating front. A minority of cases (5\%) showed subclonal staining and $19 \%$ showed a heterogeneous staining pattern. Normal cervical epithelium in the proximity of cancerous tissue was negative for L1CAM expression. Representative examples of L1CAM staining are shown in Figure 1.

Clinicopathologic characteristics in relation to L1CAM expression are shown in Table 1. The median age at time of diagnosis was 43 years (range 24-87). The majority of patients underwent a radical hysterectomy with lymph node dissection $(\mathrm{N}=347,93 \%)$; a minority underwent an abdominal hysterectomy with or without adnex extirpation $(\mathrm{N}=9,3 \%)$ or radical trachelectomy with lymph node dissection $(\mathrm{N}=16,4 \%)$. Adjuvant therapy was administered to 187 patients $(50 \%)$, of which 170 received radiotherapy and 17 received chemoradiotherapy. L1CAM expression ( $\geq 10 \%)$ was observed more frequently in HPV18 positive cases (38\%), when compared to HPV16 positive cases $(11 \%$; Odds Ratio (OR) 4.8, 95\% confidence interval (CI) $2.4-$ 9.7, $P<0.001)$. Furthermore, L1CAM expression $(\geq 10 \%)$ was observed more frequently in adenocarcinoma (37\%) and adenosquamous carcinoma (33\%) than in squamous cell carcinoma (15\%; squamous cell carcinoma vs. adeno(squamous) carcinoma: $\mathrm{OR}=3.2,95 \%$ CI $1.9-5.4$, $P<0.001)$. L1CAM expression was not associated with any of the other clinicopathologic characteristics.

\section{L1CAM expression in relation to patient survival}

Median follow-up time was 88 months (range 0-344) for all patients and 109 months (range 48-344) for the 216 patients alive at the time of data collection. Of the 120 patients that died during the follow-up period, 68 deaths could be attributed to cervical cancer. Thirty- six patients emigrated and were censored from the date of last follow-up. Of the 102 patients with disease recurrence, locoregional recurrences were observed in 45 cases, distant metastases in 48 cases and both locoregional and distant metastases in 9 cases.

Univariate Cox-regression analysis for L1CAM expression and disease-specific survival and disease-free survival is shown in Table 2. Expression of L1CAM was significantly associated with disease-free survival (Hazard Ratio (HR) $1.69,95 \%$ CI $1.10-2.60, P=0.017$, Table 2, Figure $2 \mathrm{~B}$ ). Within the subgroup of patients that did not receive adjuvant radiotherapy, the association with diseasefree survival was even stronger $(N=185, \mathrm{HR}=2.80,95 \%$ CI $1.38-5.68, P=0.004$, Supplementary Figure $1 \mathrm{~A}$ ), while the association was no longer significant in the subgroup that received adjuvant radiotherapy $(N=187, \mathrm{HR}=1.29$, $95 \%$ CI $0.74-2.25, P=0.375$, Supplementary Figure $1 \mathrm{~B})$. Subdivision of the recurrences into locoregional recurrence or distant metastasis showed that the association with disease-free survival was based on a strong association between L1CAM expression and locoregional recurrencefree survival (HR 2.86, 95\% CI $1.66-4.93, P<0.001$, Table 2 , Figure 2A). After selection of patients with free resection margins, the association between L1CAM expression and locoregional recurrences was even stronger (HR 4.10, 95\% CI $1.83-9.17, P=0.001)$. Distant metastasis-free survival was not associated with L1CAM expression (HR 0.92, 95\% CI $0.46-1.82, P=0.808$ ).

Multivariate Cox-regression analysis for L1CAM expression and established prognostic factors showed that L1CAM expression was independently associated with poor disease-specific survival (HR 1.99, 95\% CI $1.08-3.66, P=0.028$, Table 2) and L1CAM expression was the only significant prognostic factor for locoregional recurrence (HR 2.62, 95\% CI $1.33-5.17, P=0.006$, Table 2). Multivariate analysis using all prognostic factors, independent of significance in univariate analysis, showed that L1CAM expression was independently associated with disease-specific survival (HR 2.21, 95\% CI $1.12-$ $4.35, P=0.022$ ), disease-free survival (HR 1.80, 95\% CI $1.01-3.18, P=0.045)$ and locoregional recurrence-free survival (HR 2.48, 95\% CI 1.21 - 5.09, $P=0.014$ ).

Univariate Cox-regression analysis for HPV type and locoregional recurrences showed that HPV16 positive cases had significantly less locoregional recurrences, when compared to cases with other HPV types (HR 0.35, 95\% CI $0.16-0.73, P=0.006$, Figure 3A), while HPV18 positive cases (HR $0.74,95 \%$ CI $0.32-1.70, P=0.470$ ) and HPV negative cases (HR $0.69,95 \%$ CI $0.26-1.87, P$ $=0.470$ ) showed a similar risk of locoregional recurrences, when compared to cases with other HPV types. As HPV type was also associated with L1CAM expression (Table 1), stratification for HPV type and L1CAM expression was performed to ascertain whether the association between L1CAM expression and locoregional recurrences was dependent on HPV type. Stratification for L1CAM 
Table 1: Clinicopathologic characteristics in relation to $\mathrm{L} 1$ cell adhesion molecule (L1CAM) expression

\begin{tabular}{|c|c|c|c|c|}
\hline & Total & L1CAM $<10 \%$ & $\mathrm{~L} 1 \mathrm{CAM} \geq 10 \%$ & \\
\hline & $N^{\text {a }}(\%)$ & $N(\%)$ & $N(\%)$ & P-value \\
\hline \multicolumn{5}{|l|}{ Age, yrs } \\
\hline 20-35 & $92(25)$ & $67(73)$ & $25(27)$ & \\
\hline $36-45$ & $118(32)$ & $93(79)$ & $25(21)$ & \\
\hline $46-55$ & $79(21)$ & $63(80)$ & $16(20)$ & \\
\hline $56-100$ & $83(22)$ & $69(83)$ & $14(17)$ & 0.409 \\
\hline \multicolumn{5}{|l|}{ HPV type } \\
\hline Negative & $32(9)$ & $21(66)$ & $11(34)$ & \\
\hline 16 & $177(48)$ & 157 (89) & $20(11)$ & \\
\hline 18 & $58(16)$ & $36(62)$ & $22(38)$ & \\
\hline Other & $45(12)$ & $32(71)$ & $13(29)$ & $<0.001$ \\
\hline Unknown & $60(16)$ & & & \\
\hline \multicolumn{5}{|l|}{ FIGO } \\
\hline IA & $4(1)$ & $3(75)$ & $1(25)$ & \\
\hline IB & $307(83)$ & $241(79)$ & $66(21)$ & \\
\hline IIA & $52(14)$ & $41(79)$ & $11(21)$ & \\
\hline IIB & $8(2)$ & $7(88)$ & $1(12)$ & \\
\hline IIIA & $1(<1)$ & $0(0)$ & $1(100)$ & 0.397 \\
\hline \multicolumn{5}{|c|}{ Histopathology } \\
\hline $\mathrm{SCC}$ & $266(72)$ & $225(85)$ & $41(15)$ & \\
\hline AS & $12(3)$ & $8(67)$ & $4(33)$ & \\
\hline A & $94(25)$ & $59(63)$ & $35(37)$ & $<0.001$ \\
\hline \multicolumn{5}{|l|}{ Tumor size } \\
\hline$<40 \mathrm{~mm}$ & $228(61)$ & $182(80)$ & $46(20)$ & \\
\hline$\geq 40 \mathrm{~mm}$ & $121(33)$ & $92(76)$ & $29(24)$ & 0.412 \\
\hline Unknown & $23(6)$ & & & \\
\hline \multicolumn{5}{|c|}{ Infiltration depth } \\
\hline$<15 \mathrm{~mm}$ & $224(60)$ & $173(77)$ & $51(23)$ & \\
\hline$\geq 15 \mathrm{~mm}$ & $136(37)$ & $110(81)$ & $26(19)$ & 0.413 \\
\hline Unknown & $12(3)$ & & & \\
\hline \multicolumn{5}{|c|}{ Parametrial invasion } \\
\hline Negative & $316(85)$ & 247 (78) & $69(22)$ & \\
\hline Positive & $42(11)$ & $34(81)$ & $8(19)$ & 0.680 \\
\hline Unknown & $14(4)$ & & & \\
\hline \multicolumn{5}{|l|}{ Vasoinvasion } \\
\hline Negative & $153(41)$ & $116(76)$ & $37(24)$ & \\
\hline Positive & $197(53)$ & $159(81)$ & $38(19)$ & 0.268 \\
\hline Unknown & $22(6)$ & & & \\
\hline
\end{tabular}

(Continued) 


\begin{tabular}{lcccc}
\hline & Total & L1CAM $<\mathbf{1 0} \%$ & L1CAM $\geq \mathbf{1 0} \%$ & \\
\cline { 2 - 4 } & $\boldsymbol{N}^{\text {a }} \mathbf{( \% )}$ & $\boldsymbol{N}(\mathbf{\%})$ & $\boldsymbol{N}(\mathbf{\%})$ & \\
\hline Lymph nolue \\
metastasis & & & & \\
Negative & $261(70)$ & $206(79)$ & $55(21)$ & \\
Positive & $109(29)$ & $85(78)$ & $24(22)$ & 0.840 \\
Unknown & $2(1)$ & & & \\
\hline
\end{tabular}

${ }^{a}$ Total number of cases $=372$. $P$-value obtained with the Chi-square test.

HPV: human papillomavirus, FIGO: International Federation of Gynaecology and Obstetrics stage for cervical carcinoma, SCC: squamous cell carcinoma, AS: adenosquamous carcinoma, A: adenocarcinoma.

expression and HPV16 or HPV other types (HPV18, other types, HPV negative) showed that HPV16+L1CAM- cases (reference) had the lowest risk of locoregional recurrences, when compared to HPV16+L1CAM+ cases (HR 5.27, $95 \%$ CI $2.04-13.59, P=0.001)$, HPVother+L1CAMcases (HR 2.71, 95\% CI $1.24-5.90, P=0.012$ ) and HPVother+L1CAM+ cases (HR 4.89, 95\% CI $2.19-$ 10.93, $P<0.001$; Figure 3B). Histopathological type was not associated with locoregional recurrence-free survival, or any of the other survival parameters.

\section{L1CAM expression in relation to percentage of vimentin expressing tumor cells}

As L1CAM expression has been related to EMT [9, 13-16], we assessed the association between L1CAM expression and the percentage of vimentin expressing (keratin positive) tumor cells, determined by flow cytometry. A subset of 109 cases was analyzed, with a median of $13.6 \%$ of tumor cells expressing vimentin, range $2.0-95.3 \%$. Representative examples of a tumor sample showing double positive keratin/vimentin tumor cell subpopulation and a tumor sample without vimentin co-expression, with corresponding L1CAM and vimentin staining, are shown in Figure 4. Clinicopathologic characteristics in relation to the percentage of vimentin expressing tumor cells are shown in Supplementary Table 1. Percentage of vimentin expressing tumor cells was strongly associated with L1CAM expression $(P=0.003$, One-way ANOVA). Cases were subdivided into two groups, based on the $75^{\text {th }}$ percentile $(26.1 \%)$ of vimentin expressing tumor cells. For L1CAM $<10 \%$ cases, $19.5 \%$ showed high vimentin expression, compared to $45.5 \%$ of the L1CAM $\geq 10 \%$ cases. High vimentin expression was also observed more frequently in HPV 18 positive cases $(31.3 \%)$, HPV negative cases (50.0\%) and HPV other cases $(45.0 \%)$, when compared to HPV16 positive cases $(14.1 \% ; P=0.010)$. Furthermore, high vimentin expression was observed more frequently in higher FIGO stages (46.2\% in FIGO stage IIA and higher, versus $18.1 \%$ in FIGO stage IB and lower; $P=0.004)$. Percentage of vimentin expressing tumor cells was not associated with histopathological diagnosis, or any of the other clinicopathologic parameters.

For survival analysis, patients were stratified according to L1CAM expression and percentage of vimentin expressing tumor cells. The number of events in the subgroups was too low for survival analysis for locoregional recurrence-free survival. Therefore, diseasefree survival analysis was performed. L1CAM-/vimentincases (reference) had the lowest risk of recurrence, when compared to L1CAM-/vimentin+ cases (HR 1.47, 95\% CI $0.59-3.67, P=0.415), \mathrm{L} 1 \mathrm{CAM}+/$ vimentin- cases (HR 1.79, 95\% CI $0.67-4.81, P=0.247$ ) and $\mathrm{L} 1 \mathrm{CAM}+/$ vimentin+ cases (HR 3.15, 95\% CI 1.25-7.92, $P=0.015$ ).

\section{Validation cohort}

In order to validate these findings in an independent cohort, RNAseq data were used from 178 cervical cancer cases, published by the Cancer Genome Atlas Research Network $[17,18]$. L1CAM expression was divided into two groups based on median L1CAM mRNA expression (309.354, range $1-140.750 .861)$. Clinicopathologic characteristics in relation to L1CAM expression are shown in Supplementary Table 2. The median age at time of diagnosis was 46 years (range 20-88). L1CAM mRNA expression was significantly associated with fiveyear disease-free survival (HR 1.86, 95\% CI 1.08 - 3.19, $P=0.025$, Figure 2C). Furthermore, L1CAM mRNA expression was strongly correlated with the EMT score $(P<0.001)$, as determined by the level of fibronectin, $N$ cadherin, collagen-IV and PAI-1 upregulation and claudin$7, E$-cadherin and $\beta$-catenin downregulation. The EMT score has been described and validated elsewhere [17, 19].

\section{DISCUSSION}

We investigated the association between L1CAM expression and survival, as well as the association between L1CAM expression and percentage of vimentin expressing tumor cells. Our data show that L1CAM expression $(\geq 10 \%)$ was a strong independent predictor of worse locoregional recurrence-free survival in 372 
Table 2: Survival analyses for $\mathrm{L} 1$ cell adhesion molecule (L1CAM) expression

\begin{tabular}{|c|c|c|c|c|c|c|c|c|c|c|c|c|}
\hline & \multicolumn{4}{|c|}{ Disease-specific survival } & \multicolumn{4}{|c|}{ Disease-free survival } & \multicolumn{4}{|c|}{ Locoregional recurrence-free survival } \\
\hline & $N$ events & HR & $95 \% \mathrm{CI}$ & $P$-value & $N$ events & HR & $95 \% \mathrm{CI}$ & $P$-value & $N$ events & HR & $95 \% \mathrm{CI}$ & $P$-value \\
\hline \multicolumn{13}{|c|}{ L1CAM (univariate) } \\
\hline$<10 \%$ & $49(17 \%)$ & & Reference & & $73(25 \%)$ & & Reference & & $32(11 \%)$ & \multicolumn{3}{|c|}{ Reference } \\
\hline$\geq 10 \%$ & $19(24 \%)$ & 1.61 & $0.95-2.74$ & 0.077 & $22(28)$ & 1.69 & $1.10-2.60$ & 0.017 & $22(28 \%)$ & 2.86 & $1.66-4.93$ & $<0.001$ \\
\hline$<10 \%$ & & \multicolumn{3}{|c|}{ Reference } & \multicolumn{4}{|c|}{ Reference } & & \multicolumn{3}{|c|}{ Reference } \\
\hline$\geq 10 \%$ & & 1.99 & $1.08-3.66$ & 0.028 & & 1.51 & $0.91-2.49$ & 0.112 & & 2.62 & $1.33-5.17$ & 0.006 \\
\hline \multicolumn{13}{|l|}{ HPV type } \\
\hline Negative & & & & & & & & & & \multicolumn{3}{|c|}{ Reference } \\
\hline 16 & & & & & & & & & & 0.60 & $0.21-1.74$ & 0.346 \\
\hline 18 & & & & & & & & & & 1.02 & $0.34-3.03$ & 0.971 \\
\hline Other & & & & & & & & & & 1.24 & $0.40-3.88$ & 0.708 \\
\hline \multicolumn{13}{|l|}{ Tumour size } \\
\hline$<40 \mathrm{~mm}$ & & \multicolumn{3}{|c|}{ Reference } & \multicolumn{4}{|c|}{ Reference } & & \multicolumn{3}{|c|}{ Reference } \\
\hline$\geq 40 \mathrm{~mm}$ & & 2.00 & $1.13-3.54$ & 0.017 & & 2.04 & $1.29-3.22$ & 0.002 & & 1.88 & $0.98-3.59$ & 0.057 \\
\hline \multicolumn{13}{|c|}{ Infiltration depth } \\
\hline$<15 \mathrm{~mm}$ & \multicolumn{4}{|c|}{ Reference } & \multicolumn{4}{|c|}{ Reference } & & & & \\
\hline$\geq 15 \mathrm{~mm}$ & & 1.48 & $0.81-2.69$ & 0.198 & & 1.52 & $0.95-2.44$ & 0.083 & & & & \\
\hline \multicolumn{13}{|c|}{ Parametrial invasion } \\
\hline Negative & & \multicolumn{3}{|c|}{ Reference } & \multicolumn{4}{|c|}{ Reference } & & \multicolumn{3}{|c|}{ Reference } \\
\hline Negative & \multicolumn{4}{|c|}{ Reference } & \multicolumn{4}{|c|}{ Reference } & & & & \\
\hline Positive & & 1.10 & $0.56-2.18$ & 0.778 & & 1.10 & $0.65-1.86$ & 0.719 & & & & \\
\hline \multicolumn{13}{|c|}{ Lymph node metastasis } \\
\hline Negative & & \multicolumn{3}{|c|}{ Reference } & \multicolumn{4}{|c|}{ Reference } & & \multicolumn{3}{|c|}{ Reference } \\
\hline Positive & & 3.09 & $1.58-6.06$ & 0.001 & & 1.98 & $1.16-3.38$ & 0.012 & & 1.75 & $0.84-3.65$ & 0.133 \\
\hline
\end{tabular}

$\mathrm{N}=372$, L1CAM positive cases $(\geq 10 \%)=80$, L1CAM negative cases $(<10 \%)=292$.

Established prognostic factors were included in multivariate analyses if $P<0.10$ in univariate analysis. The following factors were considered: age, HPV type, histopathological diagnosis, tumour size, infiltration depth, parametrial invasion, vasoinvasion, lymph node metastasis.

* Multivariate analysis for L1CAM expression using all established prognostic factors in the model, independent of significance in univariate analysis.

$N$ events $=$ number of events $(\%), \mathrm{HR}=$ hazard ratio, $95 \% \mathrm{CI}=95 \%$ confidence interval, $\mathrm{OS}=$ overall survival, DSS $=$ disease-specific survival, DFS = disease-free survival.

cervical cancer patients (HR 2.62, 95\% CI $1.33-5.17, P$ $=0.006)$. L1CAM expression was strongly associated with percentage of vimentin expressing tumor cells $(P=0.003)$, and expression of both L1CAM and vimentin indicated a subgroup with the highest change of recurrence (HR 3.15, $95 \%$ CI $1.25-7.92, P=0.015$ ).

This is the first study reporting on the expression of L1CAM in cervical cancer specimens. L1CAM expression $(\geq 10 \%)$ was observed in $21 \%$ of cases. Studies on other gynecological cancer types reported $43 \%$ positivity in ovarian cancer (membrane + ) [8], $7-44 \%$ positivity in endometrial cancer $(\geq 10 \%)[13,20-25]$, and $16 \%$ positivity in vulvar cancer $(\geq 10 \%)$ [26]. Expression of L1CAM has been reported as an independent prognostic factor for disease-free survival in ovarian cancer [27], neuroendocrine tumors [28], gallbladder cancer [29], hepatocellular cancer [30] and endometrial cancer [20]. However, the site of recurrence (locoregional versus distant) is not specified in these studies. So far, reliable prognostic markers that can identify patients with high risk of locoregional recurrence are rare. In a cohort of 95 stage 1B cervical cancer patients undergoing radical 

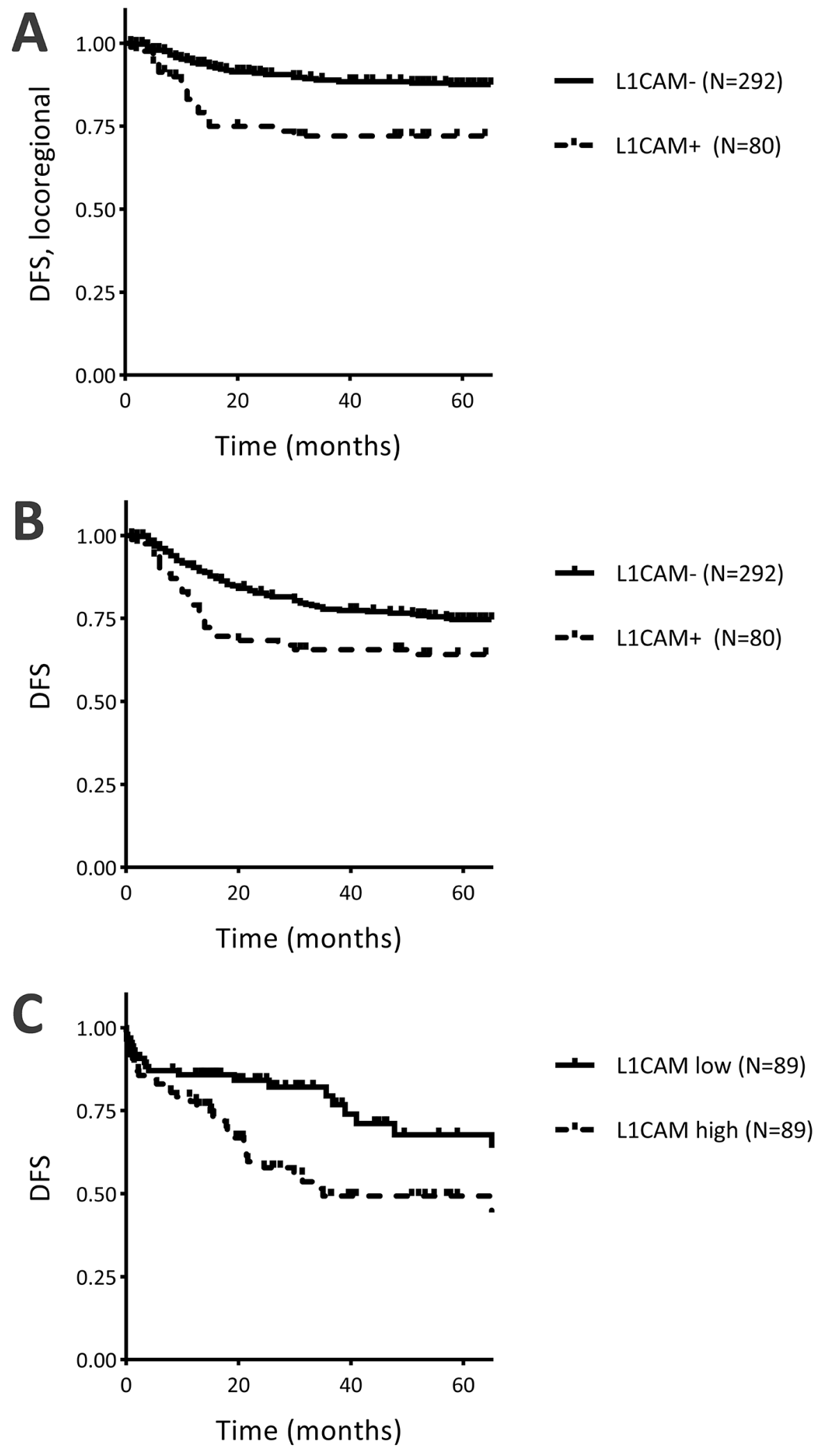

Figure 2: Survival curves for L1 cell adhesion molecule (L1CAM) expression and (A) locoregional recurrence-free survival in the research cohort; (B) disease-free survival in the research cohort; and (C) disease-free survival in the validation cohort (TCGA data). DFS = disease-free survival; L1CAM- = protein expression $<10 \%$; L1CAM $+=$ protein expression $\geq 10 \%$; L1CAM low $=$ mRNA expression below median; L1CAM high = mRNA expression above median. 
hysterectomy and pelvic lymphadenectomy, only tumor differentiation was an independent predictive factor for recurrent disease, but sole use of this feature as a criterion for adjuvant therapy would have resulted in overtreatment of low-risk patients, as almost half of patients had poorly differentiated tumors [31]. In our cohort, L1CAM expression $(\geq 10 \%)$ was observed in 80 cases, of which 22 cases $(28 \%)$ developed a locoregional recurrence, thus preselecting patients with high risk of recurrent disease, independent of the status of the resection margins. The prognostic value of L1CAM was stronger than currently established prognostic factors, such as status of resection margins and tumor size.

L1CAM might be a promising new target for antibody-based therapy in human cancers, as reviewed by Altevogt et al [32]. In mouse models for ovarian cancer, pancreatic cancer and cholangiocarcinoma, L1CAM antibodies significantly prolonged survival, reduced ascites formation and reduced tumor burden [33-36]. In our cohort, L1CAM expression was strongly associated with locoregional recurrence-free survival in patients that did not receive adjuvant radiotherapy, while the association was no longer present in the subgroup of patients that received adjuvant radiotherapy, suggesting that L1CAM positive tumors are susceptible to radiotherapy. Therefore, these results indicate that L1CAM expression might also be used to select cervical cancer patients with a high risk of locoregional recurrences, that might benefit from adjuvant radiotherapy.

As L1CAM is thought to play a role in EMT [7, 9, 13-16], we investigated the association between L1CAM expression and percentage of vimentin expressing tumor
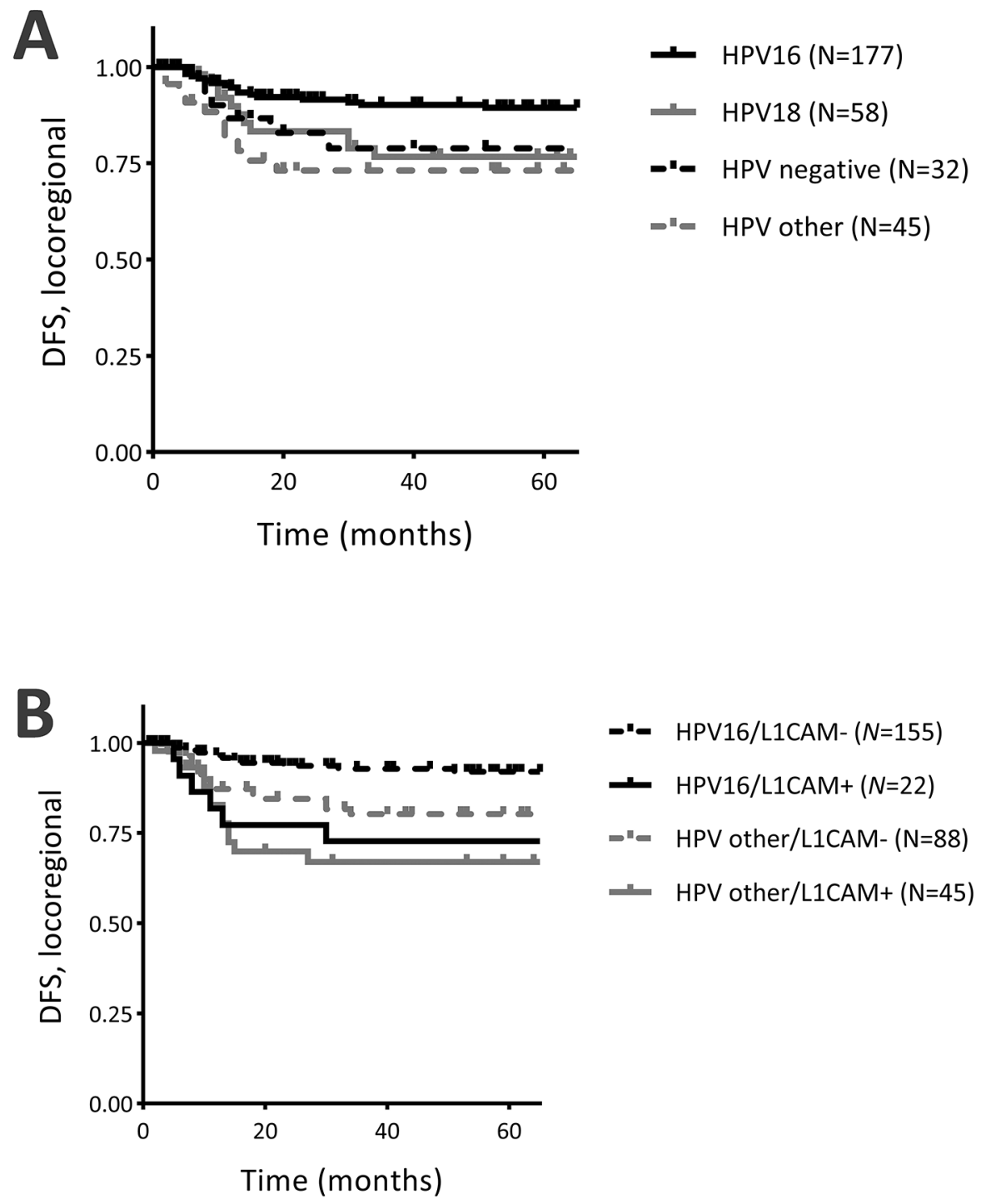

Figure 3: Survival curves for locoregional recurrence-free survival and (A) human papillomavirus type (HPV), (B) stratification for L1CAM and HPV16 / HPV other. DFS = disease-free survival; L1CAM- = L1CAM expression $<10 \%$; L1CAM $+=$ L1CAM expression $\geq 10 \%$; HPV other $=$ HPV 18 , other HPV types and HPV negative combined. 
cells, assessed by flow cytometry. L1CAM expression was strongly associated with vimentin expression. Cell line studies in human pancreatic ductal adenocarcinoma, endometrial, breast and lung cancer cells showed that treatment with TGF- $\beta 1$ initiated transcription factor slug, not only resulting in upregulated L1CAM/vimentin and downregulated E-cadherin, but also in enhanced cell invasion [9, 13-16]. Furthermore, Shtutman et al. showed that L1CAM expression leads to the disruption of adherens junctions and increases B-catenin transcriptional activity in MCF7 breast carcinoma cells [37]. In cervical cancer cell lines, such as HeLa, overexpression of L1CAM was significantly associated with differentiation. RNAi-mediated knockdown of LICAM decreased the proliferation, migration and invasion of cervical cancer cells while over-expression of L1CAM increased proliferation, migration and invasion [38].

In cervical cancer, several mechanisms are potentially involved in EMT induction, including epigenetic factors, low dose radiation, HPV oncogenes E6 and E7 and TGF- $\beta$ expression, through transcription factors twist, ZEB1, snail/slug, and several matrix metalloproteinases [39]. Tumor-associated macrophages are suggested to play an important role in the induction of EMT [40, 41]. Our cervical cancer cohort has been extensively studied, and many immune parameters are known, including the presence of infiltrating myeloid cells and their relationship to other tumor-infiltrating immune cells, tumor characteristics and patient survival
[42-44]. L1CAM expression was associated with the presence of $\mathrm{CD} 14+\mathrm{CD} 33+\mathrm{CD} 163+$ macrophages $(P=$ $0.040, \mathrm{~N}=52$, Fisher's Exact Test) and the expression of plasminogen activator inhibitor 1 (PAI-1; $P=0.010, \mathrm{~N}$ $=55$, Fisher's Exact Test). These data support published cell line studies that show that L1CAM upregulation is initiated by $\mathrm{CD} 33+$ macrophages, through TGF- $\beta 1$ in a slug-dependent fashion $[45,46]$. Understanding EMT in cervical cancer is of prime importance, as EMT leads to chemoresistance and radioresistance $[47,48]$. Based on the link found between macrophage infiltration and TGFbeta activation in the present cohort, further functional analyses are warranted and much needed to investigate the possible role of L1CAM in EMT in cervical cancer.

In our cohort, L1CAM expression was higher in adeno(squamous) carcinoma (37\%), when compared to squamous cell carcinoma ( $15 \%)$. This has also been observed in other tumor types, such as esophageal cancer [49]. Furthermore, L1CAM expression was associated with HPV type, with lower expression in HPV16 positive tumors $(11 \%)$ than in HPV18 positive tumors (38\%). In vulvar cancer, L1CAM expression was not associated with HPV [26], but little is known about the association between L1CAM and HPV infection in other cancer types. However, HPV E6/E7-transfected cervical cells showed upregulated vimentin expression and downregulated E-cadherin protein expression, suggesting a possible role for HPV in the development of EMT in cervical cancer [50]. In MDCK cells, stable expression of HPV16-E6 or
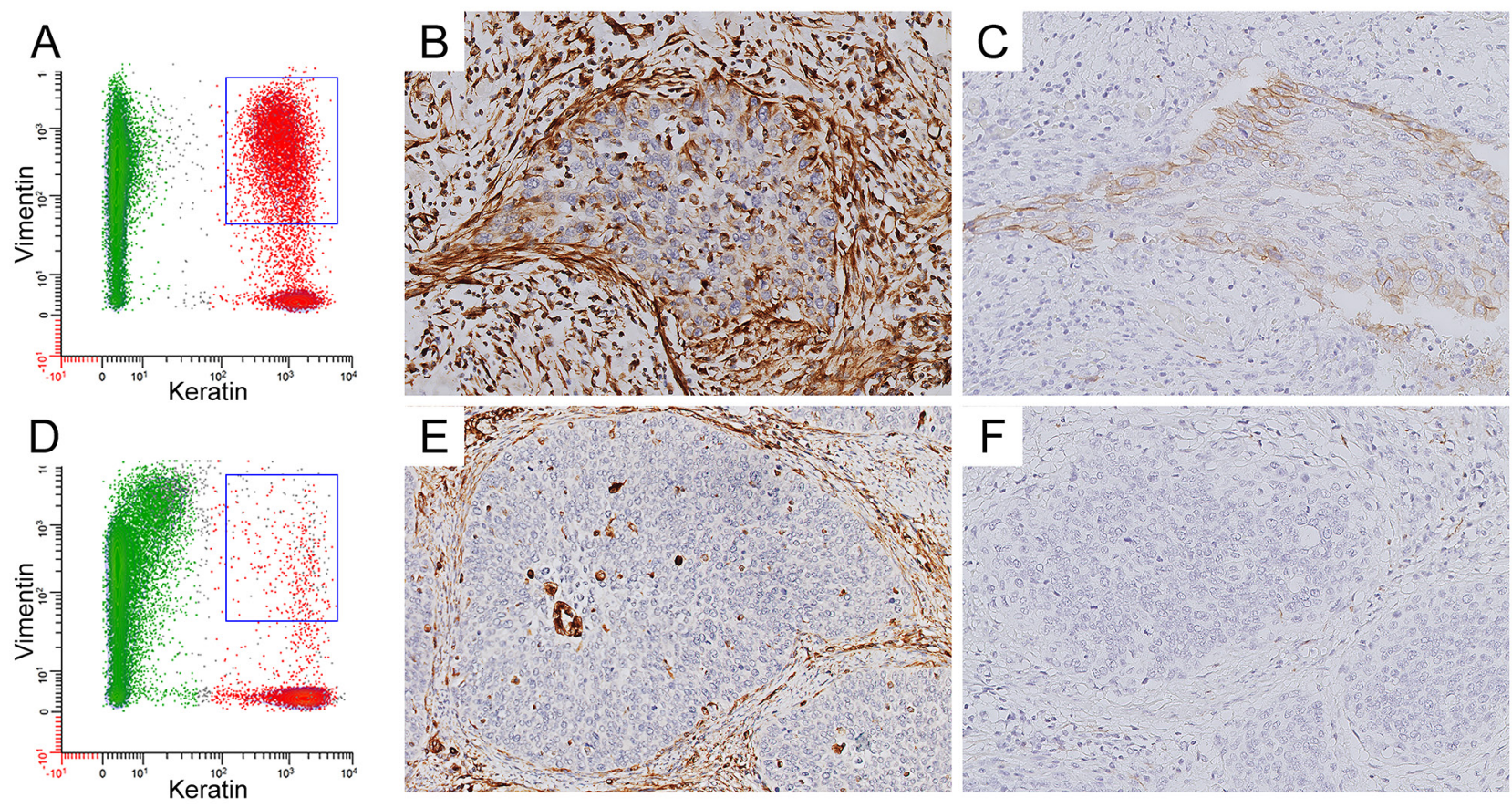

Figure 4: (A+D) Representative FACS analysis of tumor samples with high (A) and low (D) fraction of vimentin positive tumor cells. The keratin negative fraction is shown in green, the keratin positive fraction (i.e. tumor fraction) is shown in red. The keratin/vimentin double-positive tumor fraction is indicated with a blue square. (B+E) Immunohistochemical staining of high (B) and low (E) vimentin expression. (C+F): Immunohistochemical staining of high $(\mathrm{C})$ and low $(\mathrm{F})$ L1CAM expression. 
E7 induced increased expression of transcription factors slug, Twist, ZEB1 and ZEB2, accompanied with a switch from epithelial to mesenchymal markers, and a migratory and invasive phenotype [51]. A chemoresistant cervical cancer cell line (SiHaCR) showed increased levels of HPV E6 and E7 transcripts and a mesenchymal phenotype, with upregulated snail/slug/twist/vimentin and downregulated E-cadherin. Specific silencing of E6, but not E7, resulted in a more epithelial phenotype and reduced migration and invasion potential [10]. These results suggest, that the HPV E6 oncogene might play an important role in the development of EMT in cervical cancer. Further research is needed to investigate the relation between HPV and L1CAM expression.

In conclusion, L1CAM might be a promising new prognostic marker for locoregional recurrences in cervical cancer, independent of currently established prognostic markers. Furthermore, L1CAM expression was strongly associated with percentage of vimentin expressing tumor cells and expression of both L1CAM and vimentin indicated a subgroup with the highest change of recurrence, suggesting that L1CAM ascertains tumor aggressiveness, possibly through EMT.

\section{MATERIALS AND METHODS}

\section{Subjects and follow-up data}

Formalin-fixed, paraffin-embedded primary tumor samples from 372 cervical cancer patients, who underwent surgical treatment between 1985 and 2011, were retrospectively retrieved from the archives of the Department of Pathology, Leiden University Medical Center, Leiden, the Netherlands. Patients were treated according to local protocol. Patients that had received radiotherapy and/or chemotherapy prior to surgery were excluded. Clinical and follow-up data were collected through the hospital-wide oncology database. Follow-up data in this database are recorded prospectively in a deidentified manner, and are updated directly from patient medical records. Follow-up was recorded from the date of primary surgery to September 2016. Follow-up time was considered as the time in months between primary surgery and: 1) death by cervical cancer, with patients who died of a cause unrelated to cervical cancer considered as censored observations at the date of death (disease-specific survival); and 2) locoregional recurrence or distant metastasis, which ever occurred first, when both occurred within 30 days, both are stated, death is considered as a censored observation (disease-free survival).

HPV detection was performed by PCR using CP-I/ II, GP5p/6p and MY09/MY11 consensus primers. Samples that were found to be positive for HPV were subsequently sequenced to determine the HPV genotype [52]. For a part of the cohort HPV DNA was amplified using the SPF10 primer set, and HPV DNA detection and broad spectrum
HPV genotyping were performed using INNO-LiPA HPV genotyping Extra line probe assay (Innogenetics, Ghent, Belgium) [53]. Histopathological diagnosis was confirmed by review of hematoxylin-eosin slides and Periodic AcidSchiff (PAS) reagent / Alcian Blue (AB) staining by a senior pathologist. Tissue samples were used according to the guidelines of the Ethical Committee of the Leiden University Medical Center.

\section{Immunohistochemistry}

Four- $\mu \mathrm{m}$ paraffin-embedded tissue sections were deparaffinized and rehydrated. Endogenous peroxidase was blocked with $0.3 \% \mathrm{H}_{2} \mathrm{O}_{2}$ for 20 minutes. Antigen retrieval was performed in Tris-EDTA $(\mathrm{pH} 9.0)$. Slides were incubated overnight at room temperature with mouse monoclonal anti-L1CAM (1:500, IgG1, clone 14.10, BioLegend), and subsequently for $30 \mathrm{~min}$ with PowerVision-Poly/HRP (Immunologic, Duiven, the Netherlands). Immunoreactions were visualized using 0.5\% 3.30-diamino-benzidine-tetrahydrochloryde (DAB) and $0.002 \% \mathrm{H} 2 \mathrm{O} 2$ in Tris- $\mathrm{HCl}$, and counterstained with hematoxylin. The percentage of L1CAM positive tumor cells was scored independently by two authors, who were blinded to clinical data. A cut-off value of $10 \%$ was used for further analysis, as this has been shown to be the most informative cut-off. Nerve cells were used as an internal positive control.

\section{The Cancer Genome Atlas (TCGA) RNAseq data as a validation cohort}

Level 3 RSEM normalized RNA data, profiled using the Illumina HiSeq RNAseq v2, were retrieved from the TCGA data portal. Results of the TCGA RNAseq analysis have been described in detail by the Cancer Genome Atlas Research Network [17]. For our analysis, data on 178 cervical cancer patients were used, including clinical data, L1CAM mRNA expression and an EMT score. The EMT score was computed as described in the original article $[17,19]$. Briefly, the EMT score was the value resulting from the difference between the average expression of mesenchymal genes (fibronectin, $N$-cadherin, collagen-VI and $P A I-1)$ and the average expression of epithelial genes (claudin-7, E-cadherin and $\beta$-catenin).

\section{Flow cytometric analysis}

A subset of 109 FFPE cervical cancer specimens were randomly collected from the cohort stained for L1CAM by IHC, based on tumor material availability, by an investigator blinded to L1CAM expression. Of these, 22 cases $(20 \%)$ were positive for L1CAM expression based on the immunohistochemical staining described above. Flow cytometry was performed as previously described [18]. In short, cell suspensions were prepared from 2-3 dewaxed 
$60 \mu \mathrm{m}$ FFPE material sections. One million cells were incubated with $100 \mu \mathrm{l}$ of MAb mixture directed against keratin (clone MNF116, $2 \mu \mathrm{g} / \mathrm{mL}$, DAKOCytomation, Glostrup, Denmark and clone AE1/AE3, $5 \mu \mathrm{g} / \mathrm{mL}$, Chemicon International Inc, Temecula, CA, USA), and vimentin (clone V9-2b, dilution 1:5 culture supernatant (Antibodies for Research Applications, Gouda, Netherlands) overnight at $4^{\circ} \mathrm{C}$. Cells were washed and incubated with $100 \mu \mathrm{l}$ of premixed subclass-specific FITC or APC-labelled secondary reagents (Southern Biotechnology Associates, Birmingham, AL, USA). DNA was labelled with PI and RNase (Sigma-Aldrich) treated (analyzed using a FACSCalibur flow cytometer, BD Biosciences, San Jose, CA) or DAPI (analyzed using an LSRII flow cytometer BD Biosciences). A minimum of 30,000 single cell events were collected for each sample. A data file contained all events, included aggregates and debris, and data were analyzed using the WinList 8.0 and Mod-Fit 4.1 software packages (Verity Software House, Inc, Topsham, ME, USA). The fraction of keratin-positive/vimenitin-negative $(\mathrm{K}+)$ and keratin-positive/vimentin-positive $(\mathrm{V}+)$ cells was calculated after gating on single cells in the DNA-Area versus DNAWidth (doublet discrimination module) dot plot.

\section{Statistical analyses}

To assess whether L1CAM expression was associated with clinicopathologic characteristics, the chi-squared $\left(\chi^{2}\right)$ test was used. Univariate Cox-regression analysis was performed to assess the association with disease-free survival / disease specific survival. Survival curves were estimated by the Kaplan-Meier method. Multivariate Cox-regression analysis was performed to determine whether L1CAM expression was independently associated with survival, for which age, HPV type, histopathological diagnosis, tumor size, infiltration depth, parametrial invasion, vaso-invasion and lymph node metastasis were considered as covariates and included in multivariate analyses if $P<0.10$ in univariate analysis. To determine the association between L1CAM expression and percentage of vimentin expressing tumor cells, one-way ANOVA was used, as data were not normally distributed. Vimentin expression was also subdivided into two groups, based on the $75^{\text {th }}$ percentile $(26.1 \%)$ of vimentin expressing tumor cells. The association between vimentin expression and clinicopathologic characteristics was assessed using the chi-squared $\left(\chi^{2}\right)$ test. Significance tests were twosided and statistical significance was assumed when $P<0.05$, corresponding to $95 \%$ confidence intervals (CI). Statistical analyses were performed using IBM SPSS Statistics 23. This study is reported according to Reporting recommendations for tumor MARKer prognostic studies (REMARK) [54].

\section{Abbreviations}

95\% CI: 95\% confidence interval

AB: Alcian Blue
EMT: epithelial mesenchymal transition

FFPE: formalin-fixed paraffin-embedded

HPV: human papillomavirus

HR: hazard ratio

L1CAM: L1 cell adhesion molecule

NSCLC: non-small cell lung cancer

PAI1: plasminogen activator inhibitor 1

PAS: Periodic Acid-Schiff reagent

TCGA: The Cancer Genome Atlas

TGF- $\beta 1$ : transforming growth factor beta-1

\section{Author contributions}

MEV, ED and TB performed the L1CAM immunohistochemical staining; MS, MEV and TB scored the stained slides. WEC, ED and NtH performed flowcytometric analysis of vimentin expression on FFPE material. MS performed the statistical analysis. TB, CDdK, JJB, GK and ESJ provided methodological expertise. MS drafted the manuscript. All authors read, provided critical revision of and approved the final manuscript.

\section{ACKNOWLEDGMENTS}

We gratefully acknowledge Jan Molenaar, OncDoc LUMC (hospital-wide oncology database), for his help with the collection of patient follow-up data.

\section{CONFLICTS OF INTEREST}

None

\section{FUNDING}

None

\section{REFERENCES}

1. Torre LA, Bray F, Siegel RL, Ferlay J, Lortet-Tieulent J, Jemal A. Global cancer statistics, 2012. CA Cancer J Clin. 2015; 65:87-108.

2. Sedlis A, Bundy BN, Rotman MZ, Lentz SS, Muderspach LI, Zaino RJ. A randomized trial of pelvic radiation therapy versus no further therapy in selected patients with stage IB carcinoma of the cervix after radical hysterectomy and pelvic lymphadenectomy: a Gynecologic Oncology Group Study. Gynecol Oncol. 1999; 73:177-183.

3. Rotman M, Sedlis A, Piedmonte MR, Bundy B, Lentz SS, Muderspach LI, Zaino RJ. A phase III randomized trial of postoperative pelvic irradiation in Stage IB cervical carcinoma with poor prognostic features: follow-up of a gynecologic oncology group study. Int J Radiat Oncol Biol Phys. 2006; 65:169-176.

4. Samatov TR, Wicklein D, Tonevitsky AG. L1CAM: cell adhesion and more. Prog Histochem Cytochem. 2016; 51:25-32. 
5. Thies A, Schachner M, Moll I, Berger J, Schulze HJ, Brunner G, Schumacher U. Overexpression of the cell adhesion molecule L1 is associated with metastasis in cutaneous malignant melanoma. Eur J Cancer. 2002; 38:1708-1716.

6. Hua T, Liu S, Xin X, Jin Z, Liu Q, Chi S, Wang X, Wang $\mathrm{H}$. Prognostic significance of L1 cell adhesion molecule in cancer patients: a systematic review and meta-analysis. Oncotarget. 2016; 7:85196-85207. https://doi.org/10.18632/ oncotarget.13236.

7. Hung SC, Wu IH, Hsue SS, Liao CH, Wang HC, Chuang PH, Sung SY, Hsieh CL. Targeting 11 cell adhesion molecule using lentivirus-mediated short hairpin RNA interference reverses aggressiveness of oral squamous cell carcinoma. Mol Pharm. 2010; 7:2312-2323.

8. Zecchini S, Bianchi M, Colombo N, Fasani R, Goisis G, Casadio C, Viale G, Liu J, Herlyn M, Godwin AK, Nuciforo PG, Cavallaro U. The differential role of L1 in ovarian carcinoma and normal ovarian surface epithelium. Cancer Res. 2008; 68:1110-1118.

9. Tischler V, Pfeifer M, Hausladen S, Schirmer U, Bonde AK, Kristiansen G, Sos ML, Weder W, Moch H, Altevogt P, Soltermann A. L1CAM protein expression is associated with poor prognosis in non-small cell lung cancer. Mol Cancer. 2011; 10:127.

10. Vishnoi K, Mahata S, Tyagi A, Pandey A, Verma G, Jadli M, Singh T, Singh SM, Bharti AC. Human papillomavirus oncoproteins differentially modulate epithelialmesenchymal transition in 5-FU-resistant cervical cancer cells. Tumour Biol. 2016; 37:13137-13154.

11. Franklin WA, Gandara DR, Kim ES, Herbst RS, Moon J, Redman MW, Olsen C, Hirsch FR, Mack P, Kelly K. SWOG S0342 and S0536: expression of EGFR protein and markers of epithelial-mesenchymal transformation (EMT) in cetuximab/chemotherapy-treated non-small cell lung cancer (NSCLC). J Clin Oncol. 2009; 27:11076.

12. Liu S, Liu L, Ye W, Ye D, Wang T, Guo W, Liao Y, Xu D, Song H, Zhang L, Zhu H, Deng J, Zhang Z. High vimentin expression associated with lymph node metastasis and predicated a poor prognosis in oral squamous cell carcinoma. Sci Rep. 2016; 6:38834.

13. Huszar M, Pfeifer M, Schirmer U, Kiefel H, Konecny GE, Ben-Arie A, Edler L, Munch M, Muller-Holzner E, Jerabek-Klestil S, Abdel-Azim S, Marth C, Zeimet AG, et al. Up-regulation of L1CAM is linked to loss of hormone receptors and E-cadherin in aggressive subtypes of endometrial carcinomas. J Pathol. 2010; 220:551-561.

14. Geismann C, Arlt A, Bauer I, Pfeifer M, Schirmer U, Altevogt P, Muerkoster SS, Schafer H. Binding of the transcription factor Slug to the L1CAM promoter is essential for transforming growth factor-betal (TGF-beta)induced L1CAM expression in human pancreatic ductal adenocarcinoma cells. Int J Oncol. 2011; 38:257-266.

15. Kiefel H, Bondong S, Pfeifer M, Schirmer U, ErbeHoffmann N, Schafer H, Sebens S, Altevogt P.
EMT-associated up-regulation of L1CAM provides insights into L1CAM-mediated integrin signalling and NF-kappaB activation. Carcinogenesis. 2012; 33:1919-1929.

16. Lund K, Dembinski JL, Solberg N, Urbanucci A, Mills IG, Krauss S. Slug-dependent upregulation of L1CAM is responsible for the increased invasion potential of pancreatic cancer cells following long-term 5-FU treatment. PLoS One. 2015; 10:e0123684.

17. Cancer Genome Atlas Research Network; Albert Einstein College of Medicine; Analytical Biological Services; Barretos Cancer Hospital; Baylor College of Medicine; Beckman Research Institute of City of Hope; Buck Institute for Research on Aging; Canada's Michael Smith Genome Sciences Centre; Harvard Medical School; Helen F. Graham Cancer Center \& Research Institute at Christiana Care Health Services; HudsonAlpha Institute for Biotechnology; ILSbio, LLC; Indiana University School of Medicine, et al. Integrated genomic and molecular characterization of cervical cancer. Nature. 2017; 543:378-384.

18. Corver WE, Ter Haar NT, Dreef EJ, Miranda NF, Prins FA, Jordanova ES, Cornelisse CJ, Fleuren GJ. High-resolution multi-parameter DNA flow cytometry enables detection of tumour and stromal cell subpopulations in paraffinembedded tissues. J Pathol. 2005; 206:233-241.

19. Byers LA, Diao L, Wang J, Saintigny P, Girard L, Peyton M, Shen L, Fan Y, Giri U, Tumula PK, Nilsson MB, Gudikote J, Tran H, et al. An epithelial-mesenchymal transition gene signature predicts resistance to EGFR and PI3K inhibitors and identifies Axl as a therapeutic target for overcoming EGFR inhibitor resistance. Clin Cancer Res. 2013; 19:279-290.

20. Zeimet AG, Reimer D, Huszar M, Winterhoff B, Puistola U, Azim SA, Muller-Holzner E, Ben-Arie A, van Kempen LC, Petru E, Jahn S, Geels YP, Massuger LF, et al. L1CAM in early-stage type I endometrial cancer: results of a large multicenter evaluation. J Natl Cancer Inst. 2013; 105:1142-1150.

21. Bosse T, Nout RA, Stelloo E, Dreef E, Nijman HW, Jurgenliemk-Schulz IM, Jobsen JJ, Creutzberg CL, Smit VT. L1 cell adhesion molecule is a strong predictor for distant recurrence and overall survival in early stage endometrial cancer: pooled PORTEC trial results. Eur J Cancer. 2014; 50:2602-2610.

22. Van Gool IC, Stelloo E, Nout RA, Nijman HW, Edmondson RJ, Church DN, MacKay HJ, Leary A, Powell ME, Mileshkin L, Creutzberg CL, Smit VT, Bosse T. Prognostic significance of L1CAM expression and its association with mutant p53 expression in high-risk endometrial cancer. Mod Pathol. 2016; 29:174-181.

23. Smogeli E, Davidson B, Cvancarova M, Holth A, Katz B, Risberg B, Kristensen G, Lindemann K. L1CAM as a prognostic marker in stage I endometrial cancer: a validation study. BMC Cancer. 2016; 16:596.

24. Geels YP, Pijnenborg JM, Gordon BB, Fogel M, Altevogt P, Masadah R, Bulten J, van Kempen LC, Massuger 
LF. L1CAM expression is related to non-endometrioid histology, and prognostic for poor outcome in endometrioid endometrial carcinoma. Pathol Oncol Res. 2016; 22:863-868

25. van der Putten LJ, Visser NC, van de Vijver K, Santacana M, Bronsert P, Bulten J, Hirschfeld M, Colas E, Gil-Moreno A, Garcia A, Mancebo G, Alameda F, Trovik J, et al. L1CAM expression in endometrial carcinomas: an ENITEC collaboration study. Br J Cancer. 2016; 115:716-724.

26. Trietsch MD, Oonk MH, Hawinkels LJ, Bor R, van Eendenburg JD, Ivanova Z, Peters AA, Nijman HW, Gaarenstroom KN, Bosse T. Prognostic value and clinicopathologic characteristics of L1 cell adhesion molecule (L1CAM) in a large series of vulvar squamous cell carcinomas. Oncotarget. 2016; 7:26192-26205. https:// doi.org/10.18632/oncotarget.8353.

27. Bondong S, Kiefel H, Hielscher T, Zeimet AG, Zeillinger R, Pils D, Schuster E, Castillo-Tong DC, Cadron I, Vergote I, Braicu I, Sehouli J, Mahner S, et al. Prognostic significance of L1CAM in ovarian cancer and its role in constitutive NF-kappaB activation. Ann Oncol. 2012; 23:1795-1802.

28. Kim HS, Yi SY, Jun HJ, Ahn JS, Ahn MJ, Lee J, Kim Y, Cui ZY, Hong HJ, Kim JM, Li S, Hwang IG, Park K. L1 cell adhesion molecule as a predictor for recurrence in pulmonary carcinoids and large-cell neuroendocrine tumors. APMIS. 2009; 117:140-146.

29. Choi SY, Jo YS, Huang SM, Liang ZL, Min JK, Hong HJ, Kim JM. L1 cell adhesion molecule as a novel independent poor prognostic factor in gallbladder carcinoma. Hum Pathol. 2011; 42:1476-1483.

30. Guo X, Xiong L, Zou L, Sun T, Zhang J, Li H, Peng R, Zhao J. L1 cell adhesion molecule overexpression in hepatocellular carcinoma associates with advanced tumor progression and poor patient survival. Diagn Pathol. 2012; 7:96.

31. Smiley LM, Burke TW, Silva EG, Morris M, Gershenson DM, Wharton JT. Prognostic factors in stage IB squamous cervical cancer patients with low risk for recurrence. Obstet Gynecol. 1991; 77:271-275.

32. Altevogt P, Doberstein K, Fogel M. L1CAM in human cancer. Int J Cancer. 2016; 138:1565-1576.

33. Wolterink S, Moldenhauer G, Fogel M, Kiefel H, Pfeifer M, Luttgau S, Gouveia R, Costa J, Endell J, Moebius U, Altevogt P. Therapeutic antibodies to human L1CAM: functional characterization and application in a mouse model for ovarian carcinoma. Cancer Res. 2010; 70:2504-2515.

34. Arlt MJ, Novak-Hofer I, Gast D, Gschwend V, Moldenhauer G, Grunberg J, Honer M, Schubiger PA, Altevogt P, Kruger A. Efficient inhibition of intraperitoneal tumor growth and dissemination of human ovarian carcinoma cells in nude mice by anti-L1-cell adhesion molecule monoclonal antibody treatment. Cancer Res. 2006; 66:936-943.
35. Schafer H, Dieckmann C, Korniienko O, Moldenhauer G, Kiefel H, Salnikov A, Kruger A, Altevogt P, Sebens S. Combined treatment of L1CAM antibodies and cytostatic drugs improve the therapeutic response of pancreatic and ovarian carcinoma. Cancer Lett. 2012; 319:66-82.

36. Lee ES, Jeong MS, Singh R, Jung J, Yoon H, Min JK, Kim $\mathrm{KH}$, Hong HJ. A chimeric antibody to L1 cell adhesion molecule shows therapeutic effect in an intrahepatic cholangiocarcinoma model. Exp Mol Med. 2012; 44:293-302.

37. Shtutman M, Levina E, Ohouo P, Baig M, Roninson IB. Cell adhesion molecule L1 disrupts E-cadherin-containing adherens junctions and increases scattering and motility of MCF7 breast carcinoma cells. Cancer Res. 2006; 66:11370-11380.

38. Jue Zhang XW. (2016). The expression and function of L1CAM in cervical cancer--promotes tumor progression and is an independent unfavorable prognostic factor. http:// archive.jsco.or.jp/detail.php?sess_id=8548.

39. Qureshi R, Arora H, Rizvi MA. EMT in cervical cancer: its role in tumour progression and response to therapy. Cancer Lett. 2015; 356:321-331.

40. Fan QM, Jing YY, Yu GF, Kou XR, Ye F, Gao L, Li R, Zhao QD, Yang Y, Lu ZH, Wei LX. Tumor-associated macrophages promote cancer stem cell-like properties via transforming growth factor-beta1-induced epithelialmesenchymal transition in hepatocellular carcinoma. Cancer Lett. 2014; 352:160-168.

41. Helm O, Held-Feindt J, Grage-Griebenow E, Reiling N, Ungefroren H, Vogel I, Kruger U, Becker T, Ebsen M, Rocken C, Kabelitz D, Schafer H, Sebens S. Tumorassociated macrophages exhibit pro- and anti-inflammatory properties by which they impact on pancreatic tumorigenesis. Int J Cancer. 2014; 135:843-861.

42. de Vos van Steenwijk PJ, Ramwadhdoebe TH, Goedemans R, Doorduijn EM, van Ham JJ, Gorter A, van Hall T, Kuijjer ML, van Poelgeest MI, van der Burg SH, Jordanova ES. Tumor-infiltrating CD14-positive myeloid cells and CD8positive T-cells prolong survival in patients with cervical carcinoma. Int J Cancer. 2013; 133:2884-2894.

43. Hazelbag S, Kenter GG, Gorter A, Dreef EJ, Koopman LA, Violette SM, Weinreb PH, Fleuren GJ. Overexpression of the alpha $\mathrm{v}$ beta 6 integrin in cervical squamous cell carcinoma is a prognostic factor for decreased survival. $\mathrm{J}$ Pathol. 2007; 212:316-324.

44. Hazelbag S, Kenter GG, Gorter A, Fleuren GJ. Prognostic relevance of TGF-beta1 and PAI-1 in cervical cancer. Int $\mathrm{J}$ Cancer. 2004; 112:1020-1028.

45. Schafer H, Struck B, Feldmann EM, Bergmann F, GrageGriebenow E, Geismann C, Ehlers S, Altevogt P, Sebens S. TGF-beta1-dependent L1CAM expression has an essential role in macrophage-induced apoptosis resistance and cell migration of human intestinal epithelial cells. Oncogene. 2013; 32:180-189. 
46. Geismann C, Morscheck M, Koch D, Bergmann F, Ungefroren H, Arlt A, Tsao MS, Bachem MG, Altevogt P, Sipos B, Folsch UR, Schafer H, Muerkoster SS. Up-regulation of L1CAM in pancreatic duct cells is transforming growth factor beta1- and slug-dependent: role in malignant transformation of pancreatic cancer. Cancer Res. 2009; 69:4517-4526.

47. Chang L, Graham PH, Hao J, Ni J, Bucci J, Cozzi PJ, Kearsley JH, Li Y. Acquisition of epithelial-mesenchymal transition and cancer stem cell phenotypes is associated with activation of the PI3K/Akt/mTOR pathway in prostate cancer radioresistance. Cell Death Dis. 2013; 4:e875.

48. Fischer KR, Durrans A, Lee S, Sheng J, Li F, Wong ST, Choi H, El Rayes T, Ryu S, Troeger J, Schwabe RF, Vahdat LT, Altorki NK, et al. Epithelial-to-mesenchymal transition is not required for lung metastasis but contributes to chemoresistance. Nature. 2015; 527:472-476.

49. Rawnaq T, Kleinhans H, Uto M, Schurr PG, Reichelt U, Cataldegirmen G, Gawad KA, Yekebas EF, Schachner M, Izbicki JR, Kaifi JT. Subset of esophageal adenocarcinoma expresses adhesion molecule 11 in contrast to squamous cell carcinoma. Anticancer Res. 2009; 29:1195-1199.
50. Cheng YM, Chou CY, Hsu YC, Chen MJ, Wing LY. The role of human papillomavirus type 16 E6/E7 oncoproteins in cervical epithelial-mesenchymal transition and carcinogenesis. Oncol Lett. 2012; 3:667-671.

51. Jung YS, Kato I, Kim HR. A novel function of HPV16-E6/ E7 in epithelial-mesenchymal transition. Biochem Biophys Res Commun. 2013; 435:339-344.

52. Koopman LA, Szuhai K, van Eendenburg JD, Bezrookove V, Kenter GG, Schuuring E, Tanke H, Fleuren GJ. Recurrent integration of human papillomaviruses 16, 45, and 67 near translocation breakpoints in new cervical cancer cell lines. Cancer Res. 1999; 59:5615-5624.

53. Samuels S, Spaans VM, Osse M, Peters LA, Kenter GG, Fleuren GJ, Jordanova ES. Human leukocyte antigen-DR expression is significantly related to an increased disease-free and disease-specific survival in patients with cervical adenocarcinoma. Int J Gynecol Cancer. 2016; 26:1503-1509.

54. McShane LM, Altman DG, Sauerbrei W, Taube SE, Gion M, Clark GM. REporting recommendations for tumour MARKer prognostic studies (REMARK). Eur J Cancer. 2005; 41:1690-1696. 